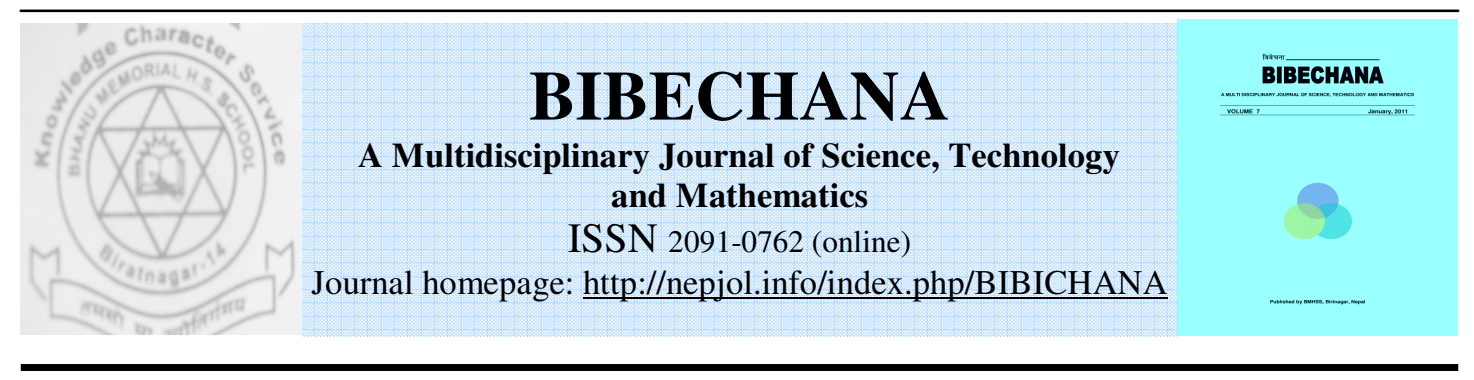

\title{
Riemann integral and its relation with Lebesgue integral
}

\author{
Mani Raj Bhattrai* \\ Dept. of Mathematics, Bhanu M.H.S. School, Biratnagar, Nepal \\ Article History: Received 29 October 2010; Revised 25 November 2010; Accepted 5 December 2010
}

\begin{abstract}
This review explains the limitations of the Riemann integral and focuses on the more general concept. It has been suggested that a better route is to abandon the Riemann integral for Lebesgue integral and explained the importance of Lebesgue integral in real analysis and other fields of mathematical science.
\end{abstract}

\section{Keywords: Riemann integral; Lebesgue integral; Fourier series}

\section{Introduction}

As part of a general movement toward rigor in mathematics in the nineteenth century, attempts were made to put the integral calculus on a firm foundation. The Riemann integral, proposed by Bernhard Riemann (1826-1866) [1], is a broadly successful attempt to provide such a foundation. Riemann's definition starts with the construction of a sequence of easily-calculated areas which converge to the integral of a given function. This definition is successful in the sense that it gives the expected answer for many already-solved problems, and gives useful results for many other problems.

In the branch of mathematics called real analysis, the Riemann integral was the first definition of the integral of a function on an interval, created by Bernhard Riemann,. While the Riemann integral is unsuitable for many theoretical purposes, it is one of the easiest integrals to define. Some of these technical deficiencies can be remedied by the Riemann-Satieties integral, and most of them disappear in the Lebesgue integral [2]. Bernhard Riemann, no doubt acquired his interest in problems connected with trigonometric series through contact with Dirichlet when he spent a year in Berlin. He almost certainly attended Dirichlet's lectures. For his Habilitationsschrift (1854) Riemann under-took to study the representation of functions by trigonometric functions.

He concluded that continuous functions are represented by Fourier series. He also concluded that functions not covered by Dirichlet do not exist in nature. But there were new applications of trigonometric series to number theory and other places in pure mathematics. This provided impetus to pursue these foundational questions. Riemann began with the question: when is a function integrable? By that he meant, when do the Cauchy sums converge?

\footnotetext{
* Corresponding author: Mani Raj Bhattrai, Dept. of Mathematics, Bhanu M.H.S. School, Biratnagar, Nepal, Email: manirb08@yahoo.co.in
} 
However, Riemann integration does not interact well with taking limits of sequences of functions, making such limiting processes difficult to analyze. This is of prime importance, for instance, in the study of Fourier series, Fourier transforms and other topics. The Lebesgue integral is better able to describe how and when it is possible to take limits under the integral sign. The Lebesgue definition considers a different class of easily-calculated areas than the Riemann definition, which is the main reason the Lebesgue integral is better behaved. The Lebesgue definition also makes it possible to calculate integrals for a broader class of functions.

In mathematics, Lebesgue integration refers to both the general theory of integration of a function with respect to a general measure, and to the specific case of integration of a function defined on a sub-domain of the real line or a higher dimensional Euclidean space with respect to the Lebesgue measure.

\section{Limitations of the Riemann integral}

Here we discuss the limitations of the Riemann integral and the greater scope offered by the Lebesgue integral. We presume a working understanding of the Riemann integral.

With the advent of Fourier series, many analytical problems involving integrals came up whose satisfactory solution required interchanging limit processes and integral signs. However, the conditions under which the integrals

$$
\sum_{k} \int_{k}(x) d x \text { and } \int\left[\sum_{k} f_{k}(x)\right] d x
$$

are equally proved quite elusive in the Riemann framework. There are some other technical difficulties with the Riemann integral. These are linked with the limit taking difficulty discussed above.

\subsection{Unsuitability for unbounded intervals.}

The Riemann integral can only integrate functions on a bounded interval. It can however be extended to unbounded intervals by taking limits, so long as this doesn't yield an answer such as $\infty \div \infty$.

\section{Domain of integration}

A technical issue in Lebesgue integration is that the domain of integration is defined as a set (a subset of a measure space), with no notion of orientation. In elementary calculus, one defines integration with respect to an orientation: $\int_{b}^{a} f=-\int_{a}^{b} f$. Generalizing this to higher dimensions yields integration of differential forms. By contrast, Lebesgue integration provides an alternative generalization, integrating over subsets with respect to a measure; this can be notated as $\int_{A} f d \mu=\int_{[a, b]} f d \mu$ to indicate integration over a subset A. For details on the relation between these generalizations, see Differential form: Relation with measures.

\section{Intuitive interpretation}

To get some intuition about the different approaches to integration, let us imagine that it is desired to find a mountain's volume (above sea level). 


\subsection{The Riemann-Darboux approach}

Divide the base of the mountain into a grid of 1 meter squares. Measure the altitude of the mountain at the center of each square. The volume on a single grid square is approximately $1 \mathrm{x} 1$ (altitude), so the total volume is the sum of the altitudes.

\subsection{The Lebesgue approach}

Draw a contour map of the mountain, where each contour is 1 meter of altitude apart. The volume of earth contained in a single contour is approximately that contour's area times its height. So the total volume is the sum of these volumes.

Folland [1] summarizes the difference between the Riemann and Lebesgue approaches thus: "to compute the Riemann integral of $f$, one partitions the domain [a, b] into subintervals", while in the Lebesgue integral, "one is in effect partitioning the range of $\mathrm{f}$ ".

The Riemann integral is a linear transformation; that is, if $f$ and $g$ are Riemann-integrable on $[\mathrm{a}, \mathrm{b}]$ and $\alpha$ and $\beta$ are constants, then

$$
\int_{a}^{b}(\alpha f+\beta g) d x=\alpha \int_{a}^{b} f(x) d x+\beta \int_{a}^{b} g(x) d x
$$

Because the Riemann integral of a function is a number, this makes the Riemann integral a linear functional on the vector space of Riemann-integrable functions. It can be shown that a real-valued function $\mathrm{f}$ on $[\mathrm{a}, \mathrm{b}]$ is Riemann-integrable if and only if it is bounded and continuous almost everywhere in the sense of Lebesgue measure. If a real-valued function on $[a, b]$ is Riemann-integrable, it is Lebesgue-integrable.

If function is a uniformly convergent sequence on $[\mathrm{a}, \mathrm{b}]$ with limit $\mathrm{f}$, then Riemann integrability of all function implies Riemann integrability of $\mathrm{f}$, and

$$
\int_{a}^{b} f d x=\int_{a}^{b} \lim _{n \rightarrow \infty} f_{n} d x=\lim _{n \rightarrow \infty} \int_{a}^{b} f_{n} d x
$$

If a real-valued function is monotone on the interval [a,b], it is Riemann-integrable, since its set of discontinuities is denumerable, and therefore of Lebesgue measure zero.

\section{Alternative formulations}

It is possible to develop the integral with respect to the Lebesgue measure without relying on the full machinery of measure theory. One such approach is provided by Daniell integral [3].

There is also an alternative approach to developing the theory of integration via methods of functional analysis. The Riemann integral exists for any continuous function $f$ of compact support defined on $\mathrm{R}_{\mathrm{n}}$ (or a fixed open subset). Integrals of more general functions can be built starting from these integrals. Let $\mathrm{C}_{\mathrm{c}}$ be the space of all real-valued compactly supported continuous functions of $\mathrm{R}$. Define a norm on $\mathrm{C}_{\mathrm{c}}$ by

$$
\|f\|=\int|f(x)| d x .
$$

Then $\mathrm{C}_{\mathrm{c}}$ is a normed vector space (and in particular, it is a metric space.) All metric spaces have Hausdorff completions, so let $\mathrm{L}^{1}$ be its completion. This space is isomorphic to the space of Lebesgue integrable functions modulo the subspace of functions with integral zero. Furthermore, the Riemann integral $\int$ is a uniformly continuous functional with respect to the norm on $\mathrm{C}_{c}$, which is dense in $\mathrm{L}^{1}$. Hence $\int$ has a unique extension to all of $\mathrm{L}^{1}$. This integral is precisely the Lebesgue integral. 
This approach can be generalized to build the theory of integration with respect to Radon measures on locally compact spaces. It is the approach adopted by Bourbaki [4] for more details see Radon measures on locally compact spaces.

\section{References}

[1] W.K.Clifford, "On the hypotheses which lie at the foundation of geometry", Nature, 8 (1873) 183.

[2] H.J. Wilcox and D.L. Myers, An Introduction to Lebesgue Integration and Fourier Series, Dover Publications, Inc., New York, (1978).

[3] P. Daniell, "A general form of integral" Ann. of Math. , 19 (1917) 279.

[4] N. Bourbaki, Elements of Mathematics, Springer, (2004). 